\title{
The role of high expectations of self and social desirability in emotional processing in individuals with irritable bowel syndrome: A qualitative study
}

\begin{abstract}
Objectives

Although high levels of distress are associated with the onset and severity of IBS, it is unclear how this relates to emotional processing, particularly in relation to maintenance of symptoms and treatment outcome. This qualitative study embedded within a randomised controlled trial aimed to explore how individuals with refractory IBS experience, express and manage their emotions after either therapist-delivered Cognitive Behavioural Therapy (TCBT) or webbased CBT (WBCBT) compared to Treatment as Usual (TAU).
\end{abstract}

\section{Design}

Cross-sectional qualitative study.

\section{Methods}

Fifty two semi-structured interviews were conducted at post-treatment with 17 TCBT participants, $17 \mathrm{WBCBT}$ and $18 \mathrm{TAU}$. The transcripts were analysed using inductive thematic analysis with grounded theory elements. NVivo 11 was used to compare themes across groups.

\section{Results}

Across all groups, high expectations of self was a recurring reason for how participants experienced and expressed their emotions. Three themes with sub-themes captured how high expectations related to specific aspects of emotional processing: perceived causes of 
emotions, strategies for coping with emotions (bottling up, avoiding emotions and active coping strategies) and the perceived interplay between emotions and IBS symptoms.

\section{Conclusions}

Patients recognised that their IBS symptoms both triggered and were triggered by negative emotions. However, there was a tendency to bottle up or avoid negative emotions for reasons of social desirability regardless of whether patients had CBT for IBS or not. Future psychological interventions in IBS may benefit from addressing negative beliefs about expressing emotions, promoting assertive emotional expression and encouraging the experience of positive emotions.

Keywords: Irritable Bowel Syndrome; Qualitative methods; Cognitive Behavioural Therapy; Emotional processing; Emotional regulation; High expectations of self. 


\section{Introduction}

Irritable Bowel Syndrome (IBS) is a chronic functional gastrointestinal disorder characterised by abdominal pain or discomfort and altered bowel patterns, with either predominantly diarrhoea, constipation or a combination of both (Spiller et al., 2007). The identification of reported symptoms through operational criteria and the exclusion of bowel organic diseases are key aspects to reaching a diagnosis of IBS (Drossman, 2006; Manning, Thompson, Heaton, \& Morris, 1978).

The prevalence of IBS ranges between $10-25 \%$ in community samples and it affects around $11 \%$ of the global population (Canavan, West, \& Card, 2014; Lovell \& Ford, 2012). Reported rates of co-morbid anxiety and depression in IBS range between 30 to $60 \%$, depending on type of health care setting and country of origin (Drossman et al., 1999; Kabra \& Nadkarni, 2013). IBS has significant financial and humanistic burdens (Nellesen, Yee, Chawla, Lewis, \& Carson, 2013; Spiller et al., 2007) and treatment relies on lifestyle advice, and medical and psychological therapies (Akehurst \& Kaltenthaler, 2001; Talley, Holtmann, \& Walker, 2015).

The biopsychosocial model of IBS acknowledges the two-way communication between mind and body (Drossman, 1998; Engel, 1980; Tanaka, Kanazawa, Fukudo, \& Drossman, 2011). This model of IBS suggests that biological and psychosocial predisposing factors in early life, such as genetics, heredity, trauma and parental illness behaviours, increase people's susceptibility to developing IBS (Deary, Chalder, \& Sharpe, 2007). Precipitating factors (e.g. lack of social support, stressful life events, gut infection) can precede and trigger IBS. Perpetuating factors, such as anxiety, depression, negative perceptions of symptoms and illness behaviours contribute to the maintenance of symptoms over time (Hauser, Pletikosic, \& Tkalcic, 2014; Spence \& Moss-Morris, 2007). 
High levels of distress are consistently associated with both the onset and maintenance of IBS (Fond et al., 2014; Henningsen, Zimmermann, \& Sattel, 2003; Sibelli et al., 2016). However, little is known about how this relates to emotional processing, particularly in relation to maintenance of symptoms and treatment outcome. The early definition of emotional processing refers to the process through which emotional disturbances are absorbed (i.e. distressed emotional reactions are changed to non-distressed or neutral reactions), allowing other experiences and behaviours to continue with minimal or no disruption (Rachman, 1980, 2001). The initial concept, conceived within a behaviourist approach, has evolved to incorporate the underlying psychological, psycho-physiological and psycho-neurological mechanisms that either promote or impede this change or "absorption". For example, cognitions can be conceived as potential inhibitors of emotional processing, including unhelpful thought patterns, negative appraisals of an event, cognitive biases and catastrophic misinterpretations (Baker, Holloway, Thomas, Thomas, \& Owens, 2004; Baker et al., 2010; Rachman, 2001). Preliminary findings suggest that individuals with IBS report stronger beliefs about the unacceptability of experiencing and expressing negative emotions compared to healthy controls and that these beliefs mediate the relationship between emotional suppression and quality of life (Bowers \& Wroe, 2016). However, the psychological mechanisms underlying emotional processing have not been sufficiently studied in IBS.

Alexithymia is a related construct that has been investigated in a handful of IBS studies. Individuals with high levels of alexithymia have a reduced ability to identify and describe feelings and a disposition to focus on external rather than internal experiences (Taylor, 1984). Alexithymia, in particular the sub-scales of difficulty with identifying and describing feelings, is positively associated with IBS symptom severity (Porcelli, De Carne, \& Leandro, 2014) and predicts poorer treatment outcome (Porcelli, De Carne, \& Leandro, 2017). 
Furthermore, one study also found poorer emotional processing in IBS (measured with the Emotional Processing Scale of Baker et al., 2010) compared to healthy controls (Phillips, Wright, \& Kent, 2013). Though preliminary findings suggest a positive correlation between poor emotional processing and IBS, further studies need to confirm its role in relation to aetiology, maintenance of symptoms and response to treatment.

The concept of emotional processing focuses on the regulation of negative emotions. However, it is also important to recognise that positive affect has been linked to lower morbidity, fewer physical symptoms, decreased pain intensity and better overall health (Pressman \& Cohen, 2005). Hence, the role of both negative and positive emotions, as well as their regulation (Gross, 2002), warrant further investigation in individuals with IBS.

Most psychological interventions for IBS follow Cognitive Behavioural Therapy (CBT) based protocols. Repeatedly, these interventions have been found to be helpful in improving treatment outcome but there is no conclusive evidence regarding the mechanisms through which CBT achieves this. Two studies found evidence that CBT had an indirect effect on IBS symptoms through anxiety and gastrointestinal specific anxiety (Jones, Koloski, Boyce, \& Talley, 2011; Ljotsson et al., 2013). However, other studies concluded that CBT had either a direct effect on IBS symptom improvement (Lackner et al., 2007) or that unhelpful behaviour and negative perceptions of IBS symptoms mediated the effects of CBT on IBS symptom severity and participation in life (Chilcot \& Moss-Morris, 2013; Reme et al., 2011). Overall, there is no definitive evidence about the role of emotional factors as mediators of treatment outcome in IBS (e.g. changing distress) and CBT studies have focused on the role of negative affect, rather than emotional processing, in the process of change in therapy.

An inductive qualitative methodology using semi-structured interviews with open ended questions allows a thorough exploration of the different aspects that either promote or impede 
successful emotional processing based on the participants' reports. Moreover, an inductive approach is likely to lead to new knowledge, capturing patients' perspectives without undue influence of existing theoretical frameworks. The current qualitative study was embedded within a multicentre randomised controlled trial (RCT) assessing the clinical and cost effectiveness of CBT in refractory IBS (i.e. ongoing symptoms after 12 months despite being offered appropriate medications and lifestyle advice) (Everitt et al., 2015). Embedding qualitative research in a clinical trial can be a useful mixed methods approach to gather complementary data on the same phenomenon (Bishop, 2015).

Facilitating aspects of emotional processing, such as emotional expression, is considered central to psychodynamic and experiential treatment approaches (e.g. emotion-focused therapy). Though CBT recognises the importance of emotions and focuses on the link between thoughts, emotions, symptoms and behaviours, emotional regulation does not tend to be its main goal (Baker et al., 2012).

The large RCT aimed to compare therapist delivered CBT (TCBT), web-based CBT selfmanagement (WBCBT) and treatment as usual (TAU) for people with refractory IBS. The objective of this nested study was to explore in depth through qualitative interviews how people with IBS experience, express and manage their emotions. Participants were interviewed soon after having received one of the three treatments. The presence of a control group allowed the researchers to draw comparisons between those participants who received CBT, via two different types of intensities, and those who did not. Patients were not interviewed during the course of treatment so as not to interfere with therapy process.

\section{Methods}

\section{Main Trial}




\section{Design.}

Five hundred and fifty-eight patients were recruited from primary and secondary care in the London and Southampton areas over two years and randomly allocated to one of the three trial groups (see Table 1 for key characteristics of the study groups and Everitt et al. (2015) for details on the CBT-based self-management programme).

\section{Nested Qualitative Study}

\section{Procedure.}

The qualitative study was approved by the relevant NRES Committee on 11 June 2013 (REC reference: 13/SC/0206). The Topic Guide of the semi-structured interviews was approved by the same committee on 4 February 2014.

At the beginning of the trial, all participants completed an online consent form and agreed to be contacted to discuss their involvement in a qualitative interview at 3 months post-baseline (i.e. post-treatment) and one at 12 months post-baseline. The current study included all 3 month interviews.

Purposive sampling was used to select for interview people with a mix of gender, age, ethnic background, geographical location (Southampton or London), study arm, symptom severity scores, and from primary and secondary care. Overall, we approached one hundred of the 558 participants in the ACTIB trial. Invitations were sent sequentially via email and 52 of the 100 invited agreed to take part; when one participant declined the invitation, another person with similar characteristics was contacted in order to achieve a final sample with a mix of clinical and demographic variables. Additional oral consent was given and recorded at the beginning of each interview. Analysis was conducted concurrently with participant selection and data collection in an iterative process. Recruitment ended when no new themes emerged from the 
analysed interviews and when each theme was thoroughly refined within a diverse sample (i.e. data saturation).

\section{Data collection.}

Fifty two participants were interviewed, 42 by the first author and 10 by an MSc student trained by the first and fifth authors. Ten interviews were face to face and 42 over the phone based on the participants' preference. Data collection lasted between September 2014 and July 2016. Interviews were digitally recorded, transcribed verbatim by a professional transcriber and anonymised to preserve confidentiality. Interviews lasted between 23 minutes to 116 minutes (mean $=56$ minutes $)$.

An interview topic guide was developed collaboratively by the research team, who have expertise in IBS and CBT for IBS. It was initially piloted with two people with IBS. The topic guide included open ended questions and prompts covering three main sections: A) participant experiences during the trial, including the effect of CBT on aspects of emotional processing (if mentioned by the participant) and emotions towards the CBT received during the study B) participant experiences with past treatments for IBS and C) emotional processing questions. Although the last section included the core questions focused explicitly on emotional processing, relevant data from all sections of the interviews were included in the analysis as some participants touched on the experience, expression and management of their emotions when asked about their IBS and their experiences during the trial (see Appendix 1 for open questions and prompts).

\section{Data analysis.}

An inductive or data driven thematic analysis of the interviews was conducted (Braun \& Clarke, 2006) with elements of grounded theory to improve the rigour of the analytical steps 
(i.e. constant comparison method and theoretical sensitivity) (Bryant \& Charmaz, 2010). Thematic analysis serves to identify, analyse and present patterns that can be found in a data set (Boyatzis, 1998), which are relevant to the description of a phenomenon associated to the research question.

The researchers worked within a contextualist constructionist epistemology, which conceives knowledge as local and situation dependent (Jaeger \& Rosnow, 1988) and sustains that individuals interpret the world within particular cultural meanings and values (Creswell, 2003). Though subjectivity is believed to be a central element of people's accounts, researchers following this epistemology aim to ground the findings on the participants' actual descriptions and triangulation is used to get a fuller picture in order to increase their validity (Madill, Jordan, \& Shirley, 2000). In the current study, a mix of perspectives from different disciplines (i.e. medicine, health psychology, CBT) and diverse cultural backgrounds enriched the interpretation of the findings by the research team.

The first step of the analysis was familiarisation with the data by reading and re-reading the first interview transcripts and noting early ideas (Dey, 1993; Joffe \& Yardley, 2004). Initial codes were developed in Word based on a close reading of the first 15 transcripts. These codes were then collated and defined to create the first draft of the coding manual, which included different divisions to enhance differentiation of codes: Code label, Description, Positive examples, Negative examples, Exceptions/restrictions (Boyatzis 1998). The first draft of the coding manual was used by the first and fourth authors independently to code the first 15 transcripts and 8 new transcripts. Coding was compared and similarities and differences discussed (between the first, fourth, fifth and last authors) before amending the coding manual to reduce ambiguities and clarify concepts. The second draft of the manual was applied by the first and fourth authors independently to 7 new transcripts and minor amendments implemented. The final coding manual was applied independently by the fourth 
author to 41 transcripts and by the first author to the whole dataset using NVivo11 software to facilitate data management and analysis.

The next steps involved: 1) collating codes into potential themes, which were broader and more abstract than codes, by undertaking interpretative data analysis (Dey, 1993) 2) reviewing the themes in relation to the codes and the entire dataset to ensure that the coding units within each theme were coherent and that the differences between each theme were clear and 3) refining and naming themes in order to generate clear definitions for each one (Braun \& Clarke, 2006). When necessary, themes were divided into sub-themes to facilitate an in-depth understanding of participants' experiences. Finally, between-group comparisons were made using NVivo 11's matrix query function. This analysis compared how people in each of the three trial groups talked about each of the themes. Finally, the written report was produced, which involved an interpretative analysis and the argument in relation to the research question. The most representative extracts of the transcripts were used as examples for each theme. For the purpose of anonymous reporting, participants were assigned pseudonyms.

\section{Results}

As shown in Table 2, the three groups had a similar number of participants and a balance of demographic and clinical variables was achieved through purposive sampling. The baseline symptom severity mean scores (Francis, Morris, \& Whorwell, 1997) suggest that on average participants had moderate symptoms of IBS.

From the interviews, it was evident that high expectations of self was an overarching theme affecting how participants experienced and expressed their emotions to others. Three themes with sub-themes captured how these high expectations played out in relation to specific aspects of emotional processing: 1) perceived causes of emotions (unrelated to IBS) 2) 
Running head: EMOTIONAL PROCESSING IN IBS

strategies for coping with emotions (sub-themes: bottling up, emotional avoidance and active coping strategies;) and 3) perceived interplay between emotions and IBS symptoms (subthemes: effects of emotions on symptoms and effects of symptoms on emotions). Figure 1 shows the thematic map of the identified themes. The relationships between themes will be unpacked in detail below.

\section{Overarching Theme 1: High Expectations of Self and Social Desirability}

Many participants talked about high expectations of self, which also had an impact on the other themes described below. These related to the different roles they assumed in their personal or professional lives. Participants also expressed critical thoughts and self-doubt regarding their performance. In some cases, they perceived that these roles had to be fulfilled to the highest level because it was expected by others.

"I would say that I'm still a little bit hard on myself sometimes and I do push myself sometimes to complete a task or - yes - get the house tidy and tea cooked before my husband gets home [...] I know that I should go and sit down, I don't: I will push myself to just finish off what I was doing [...] I've always been ... the busy one, I've always been the one that has made all the decisions and I've brought up the kids." (Abigail, Web-based CBT)

One way of talking about having high expectations of self was to construct this as a stable personality trait, affecting all aspects of the individual's life. Only participants from the CBT groups tended to use the label "perfectionism."

"So - but - um ... um being er a type of a person ... with perfectionistic tendencies, when I set myself a task, then um I go - go all out to do it [...] Um, yes um - trying to live up - to the standards that I have for myself is sometimes difficult (I: Uh huh) but I'm working on that and trying to - not um expect so much of myself." (Caroline, Web-based CBT) 
Running head: EMOTIONAL PROCESSING IN IBS

An important component of this theme reported only by female participants was the belief that one should not experience particular negative emotions, usually anger, perceiving them as unjustified or over the top.

"I sometimes find myself feeling that I'm being a bit over-the-top about things [...] Am I just being a bit of a moody-moody cow? [...] it doesn't feel like I should be feeling that way or-you know - that should be the way that I think I should be responding to something." (Caitlin, Therapist CBT)

\section{Theme 2: Perceived Causes of Emotions (Unrelated to IBS)}

Participants spoke about the causes underlying both their positive and negative emotions as having an interplay with their IBS symptoms (see Theme 4). However, they also spoke about causes independent from their IBS. A few participants in the CBT arms mentioned that therapy received during the trial was useful to identify emotional triggers, in particular cognitions underlying negative emotions (both general and illness specific).

The most commonly reported negative emotions were anxiety, low mood, sadness and frustration. The main sources of negative emotions described by participants as unrelated to their IBS can be divided in three groups: family and friends; work; and thought patterns, usually perceived as intrinsic to their personalities (see Appendix 2 for specific reasons divided by group and relevant quotes). For example, Carol from the web-based CBT arm gained some insight into how her negative emotions related mainly to her relationship with her mother.

"Actually I realised what most of my negative thoughts were about and it's mostly just about anxiety of talking to my mum, because we don't really get on that well. So if I have to ask her something or tell her something - because she usually has like a really negative reaction to everything I say." (Carol, Web-based CBT) 
Overall, perceived poor control over external situations and interpersonal conflicts (the latter due predominantly to unmet expectations and dynamics of power within the family and/or work environment) were the main factors eliciting these negative emotions.

"When my manager says things and it sort of upsets me and annoys me [...] My biggest issue in my life is with my manager and her behaviour and attitude towards people [...] one minute she's your best friend, the next minute she is shouting at you and being angry." (Joanna, Therapist CBT)

The most common labels for positive emotions reported by the participants were 'happiness' and 'positive mood'. The main sources of positive emotions were related to the quality of personal and professional relationships; free time activities or the successful performance of these activities; a sense of worth gained through helping others, through the achievement of professional goals or the external recognition of their work (see Appendix 2 for details).

"Helping somebody out, so not even thinking about it as a- a secondary action [...] see that they realise that they've been appreciated or they've been helped, that kind of makes me feel good, positive about myself [...]" (Freya, Web-based CBT)

"I got my pay rise and told my partner, that was quite a positive feeling, you know, makes you feel special and appreciated and excited." (Jason, TAU).

\section{Theme 3: Strategies for Coping with Emotions (Sub-themes: Bottling up, Emotional Avoidance and Active Coping Strategies)}

Participants spoke about spontaneous emotional expressions or automatic responses elicited when experiencing negative emotions, such as crying as a result of sadness and shouting as a result of anger or frustration. However, most participants focused on the coping strategies that they deliberately implemented when experiencing negative emotions: 1) bottling up 2) emotional avoidance 3) active coping strategies (self-care, problem solving, finding a new perspective, verbalising emotions) (see Figure 2 for details).

\section{Bottling up emotions.}




\section{Levels.}

Based on the participants' reports, three different levels of bottling up were identified: high, selective disclosure and incomplete disclosure. Individuals reporting a high level of bottling up did not tend to report using either selective or incomplete disclosure. In contrast, there was considerable overlap between selective and incomplete disclosure in that those who talked about selective disclosure tended to also use incomplete disclosure (and vice versa). The amount of disclosure depended on external factors, in particular expectations and conventions of the workplace and other social settings.

\section{High.}

Many participants reported that they usually did not disclose their emotions to others. This was mentioned in the context of anger or frustration, worry and anxiety. Some of them attributed a negative meaning to bottling up emotions, wishing they could be more capable of expressing their emotions to others.

"I internalise a lot and I don't really - tell people when I'm really worried about something [...] It's almost like a mask. I'm not lying, I just can't do it and I'm not sure why." (Mary, TAU)

\section{Selective disclosure.}

Several participants described adjusting the way they communicated their emotions depending on the person and the environment. Trust and feeling accepted and loved were the main reasons participants decided to share their emotions, usually with partners, close family and friends. In particular, for some participants it was important to withhold information about how they felt in their professional environment due to: the risk that personal situations could affect professional boundaries; and the fear of being judged by colleagues or being perceived as a difficult person or a moaner. 
"But, you know, like colleagues and people you meet, you know, on a daily basis, you don't want to be - be saying oh, I feel miserable or anything, do you? Why, what have you got to be miserable about, you know. [Laughter from P] Always moaning, you know, you don't want to do that." (Bryony, Therapist $C B T$ )

Some participants, when asked about negative emotions in general, reported talking about their IBS related worries only with specific people they trusted. Furthermore, in the context of the work environment, a few participants disclosed their IBS to specific colleagues in order to be able to cope better with their symptoms during working hours.

"I don't talk about it [IBS] with my colleagues at all [...] I just wanted it to be known by my line manager and Human Resources Department [...] so I think it was useful to know that they are aware of it so that if I do have to take days off or if I am feeling down at work, they are not just thinking, "oh [name] unreliable" or things like that." (Alastair, TAU)

\section{Incomplete disclosure.}

Some participants reported sharing their emotions or sometimes IBS related worries with others only to a certain extent, withholding specific information. The main reasons underlying this behaviour seemed to be related to the importance they attributed to the trigger of their emotions and their intensity.

"If it was just some - some silly little thing, I'll probably just keep it to myself [...] I wouldn't want to bother him [partner] with trivial things. So if it's a big thing um - then I would - I would most definitely talk to him about it." (Annabel, TAU)

"Yes, I guess it depends like if I feel particularly bad then it will be something that I need to talk about." (Pete, TAU)

\section{Reasons for bottling up emotions.}

Most participants who spoke about bottling up emotions were aware of the reasons underlying this behaviour. Some participants did not express their emotions to others because they thought it would not bring any relief, change or benefit for them. 
Running head: EMOTIONAL PROCESSING IN IBS

"Um-maybe it's not worth - talking over with anybody, particularly, you know, it's life, you have ups and downs; you've just got to get on with it. I'm a bit of a believer in just-soldiering on, you know, (I: Yes), just carrying on." (Annabel, TAU)

Others, mainly from the TAU group, decided not to express their emotions because they did not want to worry or burden other people.

"Because I don't - I don't like to burden people - because I know people have their own problems" (Samuel, TAU)

Female participants from all groups did not express their emotions because they were afraid of being rejected, judged or embarrassed by others. Most of them were afraid of being judged by people who were not close to them, in particular colleagues; however, for a few participants it was also difficult to open up with family members.

"I don't want to confront and talk to her [line manager] because I'm worried that she'll shoot me down and - embarrass me and make me feel silly." (Jane, Therapist CBT)

\section{Emotional avoidance.}

Many participants performed tasks, activities or chores to take their mind off their negative emotions or the thoughts leading to these emotions. The forms of avoidance and distraction varied greatly. Some listened to music, others read a book, exercised or used therapeutic strategies such as worry time.

"I try and distract myself by thinking of other things. Sometimes they work and sometimes they don't. And one of the things I do, which probably sounds really bizarre, is to put counties of England into alphabetical order because - or to go around the country, trying to position all the counties." (Cressida, Web-based CBT)

\section{Active coping strategies.}

Though bottling up and emotional avoidance were predominant coping strategies, several participants from all groups described implementing active coping strategies to manage their 
negative emotions, which they felt were helpful (see Figure 2 and Appendix 3 for descriptions and patient quotes).

Two main differences were identified between the CBT groups and the TAU group. Firstly, only participants from the CBT arms described their personalities as strong or resilient, which seemed to be the main reason they used problem solving as a coping strategy.

"I think it's just when I feel very negatively about something, I actually do something to correct it. So I'm not someone who just accepts the situation; I've got quite like a strong fighting personality, so so when things get too negative, then I just - I just change them." (Eva, Therapist CBT)

Secondly, participants from the CBT groups tended to describe in more detail the cognitive processes that allowed them to reframe a situation causing negative emotions (e.g. doing a pros and cons list, replacing their negative thoughts for more realistic ones).

"I've got control over that and I can change the way I am towards it. So instead of getting really stressed and worked up about things, I can look at alternative thoughts and alternative ways of looking at something." (Joanna, Therapist CBT)

\section{Theme 4: Perceived interplay between emotions and IBS symptoms}

\section{Effects of emotions on physical symptoms.}

Many participants perceived anxiety and worry as the main triggers of their IBS symptoms. Participants also expressed that the reduction in the intensity of these negative emotions improved their physical symptoms. While some participants described in detail the family or work related situations triggering their IBS symptoms, others spoke about general emotions feeding them.

"I can really feel my stomach kind of muscles tensing when I'm really upset or if I'm anxious or worried about something [...] and I can make myselffeel ill, you know, if I think it in my head [...] I know that my emotions do affect my stomach." (Jasmine, TAU) 
Participants also mentioned the helpful effect that positive emotions, such as happiness, had on their bowel functioning. For example, Barbara from the Web-based CBT group described positive emotions as "buffers" of her symptom severity.

"So there's a really - I can't work out that relationship with it and I guess it depends on perhaps how - high my mood is, depends on how much of a buffer it can act in terms the physical symptoms trying to annoy me." (Barbara, Web-based CBT)

Interestingly, some participants talked about their bowels as an "organ that can feel emotions", highlighting the significant effect that their emotions have on their bowels.

"So I know if I can remain positive up there, it will have a positive impact on here. So - pointing at the stomach [...] As I said, I know the stomach will be happier if I'm happier, then I know it has a part." (Angela, TAU)

\section{Effects of physical symptoms on emotions.}

Participants talked about how their IBS symptoms had a direct negative impact on their mood. Furthermore, the absence of symptoms or an improvement in their severity seemed to act as mood boosters. Anxiety and worry were the most common emotions reported as consequences of IBS symptoms.

"If I've got an IBS flare-up, I'll notice kind of more negative feelings creeping in." (Holly, Therapist $C B T)$

Participants linked different emotional states to particular IBS related changes. Anxiety seemed to be associated with the unpredictability of symptoms throughout the day, which restricted participants' social life as well as their work performance; and to the potential scenario of not being able to cope with an accident.

"I get anxious a little bit when I'm out, yes, I still - that hasn't completely gone yet. I do get anxious because if I'm on my feet for too long, that's when it can set it off [...] And sometimes we do have to literally just drop everything and come home, you know, because I need to go to the toilet." (Abigail, Web-based CBT) 
Some participants reported feeling low, down, hopeless or depressed when their symptoms flared up or got worse. These emotions seemed to be particularly relevant when IBS symptoms came back unexpectedly taking participants back to the reality of living with a chronic condition and making them feel disempowered.

"When symptoms - play up, like they did yesterday, it worries me how much it really puts me down and I can be really quite down but it certainly really takes a hit on my mood and general well-being feelings, sort of thing, when they do play up. (I: Okay) It's not-and I don't like how much it does get to me." (Barbara, Web-based CBT)

Many participants spoke about feeling frustrated, fed up, annoyed or angry as a consequence of having IBS. These emotions seemed to be associated with the perception that their body had failed them and they could not lead a "normal life" compared to others or to their "old self"; the belief that they were not able to manage their symptoms successfully; and the perception that other people did not understand the real impact of IBS.

"It was just sort of - you feel like your body's let you down. Other people don't experience these things [...] whereas you-you feel, to some extent, being held back and that you can't, you know, like the life that you had before was a lot more relaxed and didn't have this stress. So it's only natural to feel angry about that." (John, TAU)

\section{Discussion}

\section{Summary of the Results}

To our knowledge, this is the first study that explored in depth how individuals with IBS experience, express and manage their emotions. The analysis highlighted that beliefs around high standards of self and social desirability seem to be important elements shaping the way participants experienced their emotions as well as the way they decided to express/not express them. This was captured by three main themes: perceived causes of emotions (unrelated to IBS), strategies for coping with emotions and the interplay between emotions 
Running head: EMOTIONAL PROCESSING IN IBS

and IBS symptoms (see Figure 3 for a proposed model summarising the relations between themes).

The theme around the interplay between emotions and IBS helped to clarify how participants perceived the complex interactions between their emotional states and their physical symptoms. Anxiety was described both as a trigger and a consequence of IBS symptoms while the rest of the reported negative emotions (frustration and low mood) were perceived mainly as consequences of symptoms. On the other hand, positive mood and happiness were labelled by participants as buffers of symptom severity. Moreover, Figure 3 shows that participants also described positive and negative emotions unrelated to IBS, linked to other general aspects of their life. In turn, these emotions unconnected to IBS were perceived to have a positive/buffer effect or a negative impact on IBS symptoms.

Although several participants mentioned the use of active coping strategies, many across all groups bottled up emotions and used emotional avoidance to cope with negative emotions. Adding to previous research exploring the potential role of emotional suppression in IBS (Ali et al., 2000), the current study revealed that bottling up is not perceived as an all-or-nothing strategy but can be applied selectively depending on context and on the underlying motivations of each individual. Selective and incomplete disclosure can be adaptive coping strategies. However, participants using these coping strategies tended to express a wish to be able to communicate their emotions to more people or share emotional information with fewer filters.

Interestingly, alexithymia did not appear as a theme in the current study. This may be associated with the use of semi-structured interviews, which allowed prompts to promote conversation flow and explore how participants recognised and talked about emotions (Fava, Freyberger, \& Bech, 1995). Furthermore, it may be that the present sample showed more 
Running head: EMOTIONAL PROCESSING IN IBS

emotional awareness and openness to talk about their emotional experiences due to their willingness to take part in a study offering psychological therapy for their IBS. Finally, it may be related to the nature of the patients' presenting problems (i.e. that they did not have this specific problem. We cannot assume that it is there but not discussed).

\section{Group Differences in Emotional Processing}

For some participants, the CBT offered during the trial seemed to improve their understanding of emotional triggers. Furthermore, only participants from the CBT groups tended to describe their personalities as strong or resilient when talking about problem solving emotions, suggesting that they felt more empowered than the TAU group. A sense of empowerment to manage IBS symptoms was a predominant pattern reported by the CBT participants when asked about their experiences of the main trial. The way CBT participants talked about a perceived increased control over their IBS symptoms, gained through new selfmanagement techniques learned during the study, may have had an impact on the way they subsequently identified and described active coping strategies related specifically to managing their negative emotions. Furthermore, emotional regulation may have been addressed with some individuals if it arose as a pertinent issue in the therapy sessions.

Participants receiving CBT also explained in more detail the analytical processes which allowed them to reframe a situation. Some explicitly acknowledged the positive role CBT played in changing their thought processes. Despite these promising group differences, there was still a clear need to address unhelpful emotional coping strategies and deep seated high expectations of self in all groups. Bakers and colleagues (2012) found that patients referred by their GPs to NHS psychological services, primarily due to anxiety or depression symptoms, showed significant improvement in emotional processing after receiving CBT. 
Running head: EMOTIONAL PROCESSING IN IBS

Further studies are needed to explore if CBT has an impact on emotional processing specifically in IBS and to unpack its mechanisms of change.

\section{High Standards of Self and Social Desirability}

Hewitt and colleagues have proposed a multi-dimensional model of perfectionism which includes high standards of self, critical self-evaluation, standards imposed on other individuals and standards perceived to be imposed by others (Hewitt, Flett, Besser, Sherry, \& McGee, 2003). Although some participants in the present study spoke about negative emotions elicited by high standards imposed on others and by others, most of them focused on high standards of self and critical self-evaluation, particularly in relation to performance of family and professional roles, unacceptability of experiencing and expressing negative emotions, and perceived poor control both in terms of coping with negative emotions and IBS symptom management. All these areas included critical thoughts, "should \& oughts" and selfdoubt statements. In line with these findings, a recent study suggested that maladaptive perfectionism promoted self-critical thoughts, which in turn lead to self-blame as a coping mechanism for stress (Sirois \& Molnar, 2014). Furthermore, self-critical perfectionism has been associated with the loss of resilience of the stress system caused by "wear and tear" of chronic stress (Kempke, Luyten, Mayes, Van Houdenhove, \& Claes, 2016). More focus on addressing beliefs about high standards of self and social desirability in people with IBS may be a valuable path to decrease stress levels and reduce its negative physiological effects in the short and long term. Though we found that male participants did not report "should or oughts" in relation to their emotional experiences and they did not talk about fear of rejection as a reason for bottling up, these preliminary findings should be explored in the future with IBS male samples. 
The participants' perception of poor control over symptoms and emotional experiences was an important source of negative emotions whereas their sense of worth (increased through the achievement of professional/personal goals and the recognition of their work by others) elicited positive emotions. Similarly, Grodzinsky and colleagues reported higher levels of negative self-esteem and lower levels of sense of coherence (i.e. an enduring and dynamic feeling of confidence that allows an individual to choose appropriate coping strategies depending on the situation) in an IBS sample compared to individuals with Inflammatory Bowel Disease (Antonovsky \& Sagy, 1986; Grodzinsky et al., 2015). Therefore, focusing on self-concept, self-esteem and self-efficacy may represent a more integrative approach to promote patients' well-being through the experience of more positive emotions, more sense of control over IBS symptoms and over negative emotions, and reduction of self-doubt.

\section{Bottling up and Emotional Avoidance vs Active Coping Strategies}

The suppression of negative emotions may eventually lead to emotional distress (Hawkins \& Cougle, 2011); therefore, addressing views about the unacceptability of experiencing and expressing negative emotions may decrease distress and improve the patients' overall wellbeing. More importantly, these are modifiable elements during therapy that can elicit answers about behaviours which can be more automatic for the individual (Rimes \& Chalder, 2010).

Participants in the current study reported that different or conflicting communication styles triggered negative emotions. Negative social interactions seem to be a stronger cause of psychological distress than low social support (Lackner et al., 2013) and seem to have a stronger effect than positive interactions on physical health because they elicit negative emotions, which in turn release physiological effects (De Vogli, Chandola, \& Marmot, 2007). Hence, addressing interpersonal aspects that both obstruct and promote assertive communication may decrease distress levels in patients with IBS. Finally, introducing the 
idea that individuals can work towards a more open communication of their emotions in safe environments may help to address the belief that bottling up is a stable behaviour that cannot be changed.

Whilst some studies have reported that individuals with IBS tend to use less active coping strategies (Knowles et al., 2016) and more emotion oriented coping (Drossman et al., 2000) compared to controls, Phillips and colleagues (2013) concluded that people with IBS seek more instrumental support and implement more problem solving. Though the findings of this qualitative study supported the key role of bottling up and emotional avoidance, participants across the three groups still reported the use of active coping strategies, which were sometimes associated with a positive self-concept. Future studies focused specifically on emotional coping (not only symptom management) are needed to explore this further.

\section{Clinical Implications and Future Research}

As shown in Figure 3, the findings of this qualitative study suggest that psychological interventions in IBS may benefit not only from addressing the illness related causes of negative emotions but also other aspects of the patients' lives which are perceived as key triggers of psycho-emotional distress. The main areas seem to be resolving communication problems and conflicts within the family and work environments, reappraising expectations about themselves and others and promoting sense of control over negative emotions. Furthermore, positive emotions seem to act as buffers of IBS symptoms and interventions may improve their effectiveness by increasing the patient's social network and improving the quality of relationships with family and colleagues; promoting a sense of pride and satisfaction in relation to more realistic professional and personal goals; encouraging behaviours congruent to their own life values (such as helping others); and hobbies/free time activities. Research suggests that positive affect may promote health by buffering the harmful 
effects of stress through the promotion of resilience, endurance and restorative activities, such as exercise and sleep (Pressman \& Cohen, 2005; Robles, Brooks, \& Pressman, 2009). Overall, the regulation of both negative and positive emotions, rather than processing negative emotions only, seems to impact on the maintenance and management of IBS symptoms.

It still remains important to address IBS related triggers of negative emotions during interventions by reinforcing sense of control in terms of symptom management, promoting the acceptance of a chronic and relapsing condition as well as of the feelings of loss regarding the "old self" or the "old normal life", encouraging disclosure of IBS symptoms and related worries in safe environments to increase social support to cope better with the condition.

It is worth noting that participants referred to their stomachs/bowels as a feeling/sensing part of themselves, a novel finding that has not been sufficiently studied in previous IBS literature. Future qualitative studies focused on the embodied experience of individuals with IBS could enrich the understanding of how IBS affects patients' sense of their own body.

The current study has some limitations. Qualitative findings cannot be generalised and participants had volunteered to be in a CBT trial, which may make them systematically different to other IBS patients. As described above, individuals from the CBT groups may have been primed to discuss emotional active coping because of the context of the interview itself after having received CBT. Furthermore, the sample was biased towards White British females, which was reflective of the sample recruited to the main trial. We conducted comparisons between participants from a white ethnic background vs participants from all other backgrounds. Interestingly, only white participants reported bottling up emotions because they did not want to worry others or to avoid confrontation. Previous research has found that culture can have indirect effects via parental socialisation on the way people 
identify and verbalise emotions (Le, Berenbaum, \& Raghavan, 2002). Further studies are needed to provide a better insight into how ethnicity and cultural values play a role in the experience and expression of emotions amongst IBS patients. It would also be pertinent to conduct qualitative comparative studies with an IBS sample and healthy controls with similar demographic characteristics.

The study has several methodological strengths which can facilitate the transferability of findings to similar contexts: strong purposive sampling, inclusion of patients both from primary and secondary care, robust audit trail, good coding rigour and multiple perspectives from different disciplines in data interpretation. Future mixed methods research should study further the role of emotional processing in IBS in relation to its aetiology and maintenance of symptoms and the role of different emotional factors as predictors and moderators of treatment outcome.

In conclusion, high standards of self and social desirability seem to be important aspects shaping the way individuals with IBS experience, express and manage their emotions. Future psychological interventions in IBS may benefit from addressing negative beliefs about experiencing and expressing negative emotions, promoting assertive expression of emotion, exploring the negative effects of emotional avoidance, encouraging the experience of positive emotions and improving a sense of control in terms of emotional coping. Promoting the regulation of both positive and negative emotions may reduce the negative effects of psychological distress on IBS symptoms, increase the patients' overall well-being in the short and long term and in turn improve physical outcomes. 
Running head: EMOTIONAL PROCESSING IN IBS

\section{Tables}

Table 1

Brief description of the three trial groups

\begin{tabular}{llll}
\hline \multicolumn{1}{c}{ Therapist CBT } & \multicolumn{1}{c}{ Web-based CBT self- } & Treatment as usual \\
& management programme & \\
\hline Manualised CBT based on & 8 CBT based online modules & Usual GP or consultant care \\
paper manual & $3 \times 30$ minute therapist & Leaflet with NICE \\
6 x 60 minute telephone & telephone sessions during 12 & recommendations for IBS to \\
sessions over 12 weeks & weeks & participants \\
Booster sessions at 4 and 8 & Booster sessions at 4 and 8 & NICE guidelines for IBS to \\
months & months & GPs \\
Usual GP or consultant care & Usual GP or consultant care &
\end{tabular}

Note. The content of the CBT programme is similar in both active arms of the trial but the delivery vehicle differs (i.e. paper manual in the Therapist CBT group and website in the Web-based CBT self-management group). 
Running head: EMOTIONAL PROCESSING IN IBS

Table 2

Demographic and clinical characteristics of each trial group and overall sample

\begin{tabular}{|c|c|c|c|c|}
\hline & $\begin{array}{c}\text { Therapist } \\
\text { CBT } \\
(n=17)\end{array}$ & $\begin{array}{c}\text { Web-based } \\
\text { CBT } \\
(n=17)\end{array}$ & $\begin{array}{c}\text { TAU } \\
(n=18)\end{array}$ & $\begin{array}{l}\text { Total Sample } \\
\quad(n=52)\end{array}$ \\
\hline $\begin{array}{l}\text { Gender } \\
\mathrm{N}(\%)\end{array}$ & $\begin{array}{c}\text { Females }=13 \\
(76.5 \%)\end{array}$ & $\begin{array}{c}\text { Females }=14 \\
(82.4 \%)\end{array}$ & $\begin{array}{c}\text { Females }=13 \\
(72.2 \%)\end{array}$ & $\begin{array}{c}\text { Females }=40 \\
(76.9 \%)\end{array}$ \\
\hline Ethnicity & White-British $=11$ & White-British $=12$ & White-British $=15$ & White-British $=38$ \\
\hline $\mathrm{N}(\%)$ & $\begin{array}{c}\text { White Other=4 } \\
\text { White-Asian=1 } \\
\text { African=1 }\end{array}$ & $\begin{array}{c}\text { White Other=4 } \\
\text { Other-ethnicity=1 }\end{array}$ & $\begin{array}{c}\text { White-Other }=1 \\
\text { Indian }=1 \\
\text { Irish }=1\end{array}$ & $\begin{array}{c}\text { White-Other=9 } \\
\text { White-Asian=1 } \\
\text { African=1 } \\
\text { Indian=1 } \\
\text { Irish=1 } \\
\text { Other-ethnicity=1 }\end{array}$ \\
\hline Age & 39.94 & 42. & 39.72 & 40.67 \\
\hline Mean (SD) & (11.71) & $(17.37)$ & (13.23) & (14.06) \\
\hline IBS-SSS score & 283.47 & 259.65 & 236.83 & 259.54 \\
\hline Mean (SD) & (117.11) & (124.39) & (86.36) & $(109.61)$ \\
\hline $\begin{array}{l}\text { Recruitment } \\
\text { site } \\
\mathrm{N}(\%)\end{array}$ & $\begin{array}{l}\text { Primary care }=11 \\
\text { Secondary care }=6\end{array}$ & $\begin{array}{l}\text { Primary care }=13 \\
\text { Secondary care }=4\end{array}$ & $\begin{array}{l}\text { Primary care }=11 \\
\text { Secondary care }=7\end{array}$ & $\begin{array}{c}\text { Primary care }=35 \\
\text { Secondary } \\
\text { care }=17\end{array}$ \\
\hline $\begin{array}{l}\text { Duration of } \\
\text { symptoms in } \\
\text { years before } \\
\text { study entry } \\
\text { Mean (SD) }\end{array}$ & $\begin{array}{l}15.25 \\
(7.3)\end{array}$ & $\begin{array}{l}15.52 \\
(9.04)\end{array}$ & $\begin{array}{c}17.92 \\
(12.89)\end{array}$ & $\begin{array}{l}16.26 \\
(9.96)\end{array}$ \\
\hline
\end{tabular}




\section{Figures}

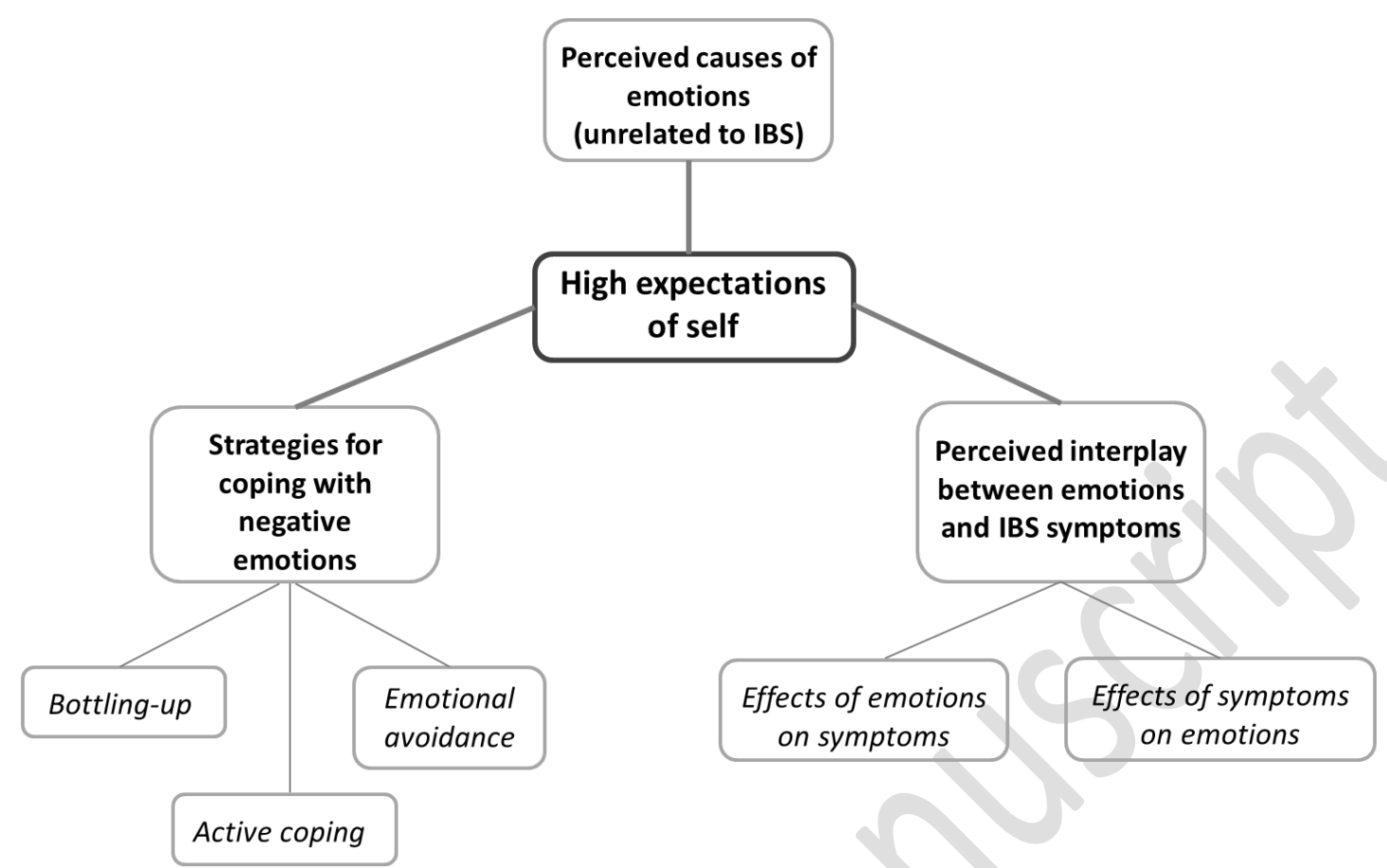

Figure 1. Thematic map showing the themes (bold) and sub-themes (italics) identified during the analysis. One overarching theme (High expectations of self) and 3 further themes with their respective sub-themes captured the participants' reports in relation to their emotional experience, emotional expression and emotional coping. 


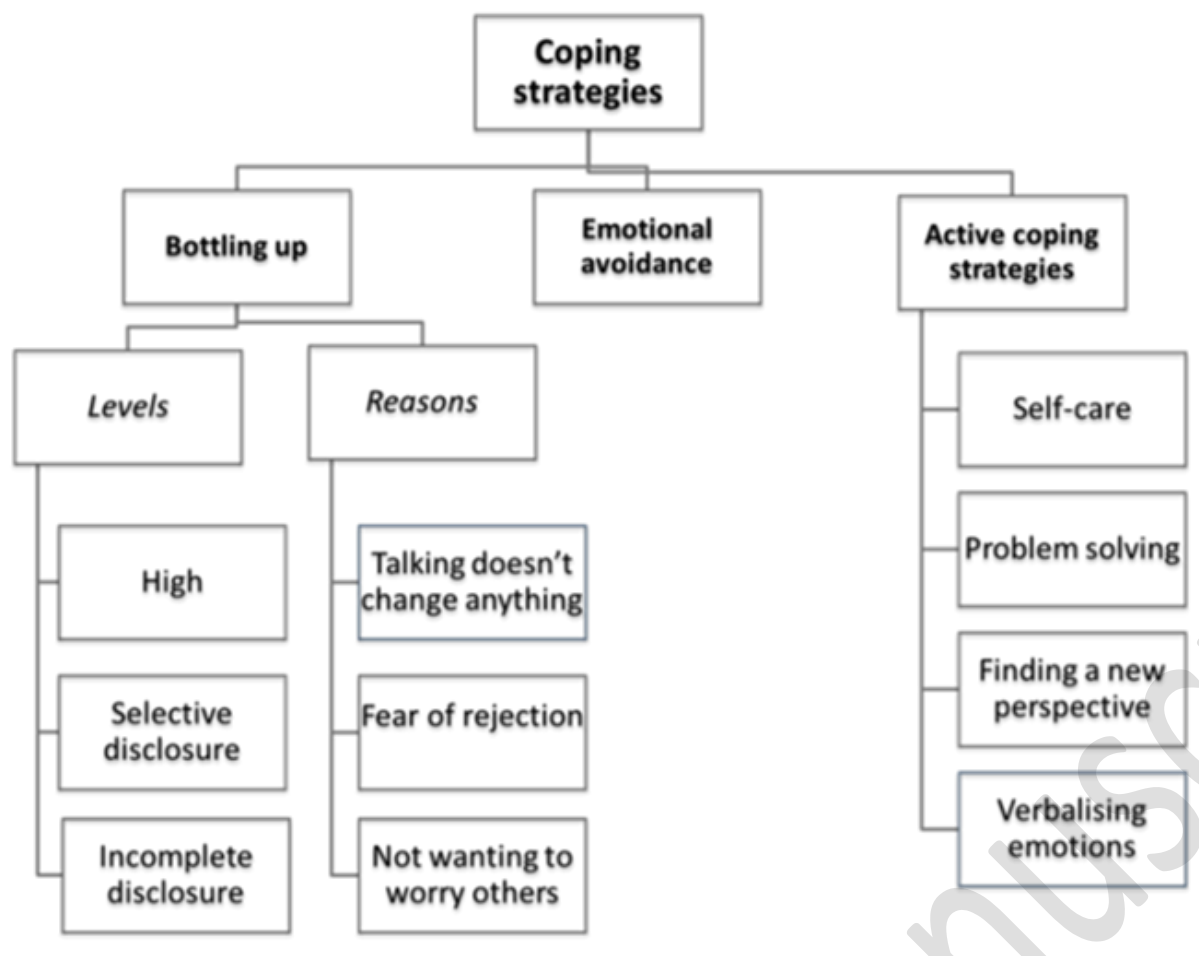

Figure 2. This diagram illustrates the different strategies for coping with emotions described by the participants. All levels of bottling up relate to the three main reasons. 


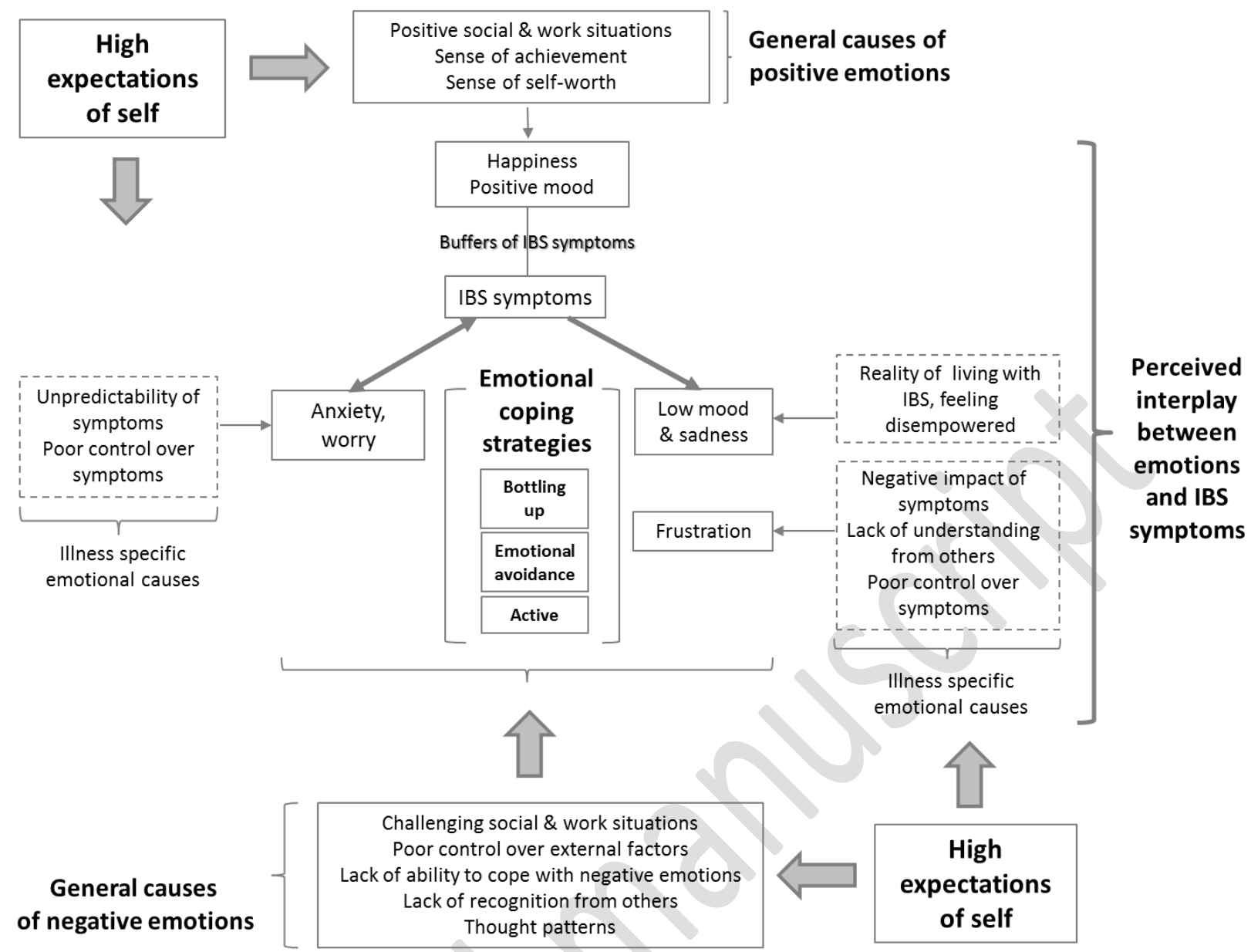

Figure 3. Proposed model illustrating the relationships between emotions, emotional triggers and IBS symptoms based on the participants' reports. High expectations of self seem to be recurrent reasons underlying both the general and illness related causes of negative emotions. These expectations also lead to positive emotions (perceived as buffers of symptoms) when they are associated to a sense of achievement and self-worth. The central part of the diagram shows the perceived interplay between emotions (positive and negative) and IBS symptoms. Three types of emotional coping strategies are used to cope with the most predominant negative emotions reported by the participants. Themes are shown in bold: High expectations of self (overarching theme); General causes of emotions (unrelated to IBS); Emotional coping strategies; Perceived interplay between emotions and IBS symptoms (which includes illness specific emotional causes). 


\section{References}

Akehurst, R., \& Kaltenthaler, E. (2001). Treatment of irritable bowel syndrome: a review of randomised controlled trials. Gut, 48(2), 272-282.

Ali, A., Toner, B. B., Stuckless, N., Gallop, R., Diamant, N. E., Gould, M. I., \& Vidins, E. I. (2000). Emotional abuse, self-blame, and self-silencing in women with irritable bowel syndrome. Psychosom Med, 62(1), 76-82.

Antonovsky, H., \& Sagy, S. (1986). The development of a sense of coherence and its impact on responses to stress situations. J Soc Psychol, 126(2), 213-225.

Baker, R., Holloway, J., Thomas, P. W., Thomas, S., \& Owens, M. (2004). Emotional processing and panic. Behav Res Ther, 42(11), 1271-1287. doi: 10.1016/j.brat.2003.09.002

Baker, R., Owens, M., Thomas, S., Whittlesea, A., Abbey, G., Gower, P., .. Thomas, P. W. (2012). Does CBT facilitate emotional processing? Behav Cogn Psychother, 40(1), 19-37. doi: $10.1017 /$ S1352465810000895

Baker, R., Thomas, S., Thomas, P. W., Gower, P., Santonastaso, M., \& Whittlesea, A. (2010). The Emotional Processing Scale: scale refinement and abridgement (EPS-25). J Psychosom Res, 68(1), 83-88. doi: 10.1016/j.jpsychores.2009.07.007

Bishop, F. L. (2015). Using mixed methods research designs in health psychology: An illustrated discussion from a pragmatist perspective. British Journal of Health Psychology, 20(1), 5-20. doi: 10.1111/bjhp.12122

Bowers, H., \& Wroe, A. (2016). Beliefs about emotions mediate the relationship between emotional suppression and quality of life in irritable bowel syndrome. J Ment Health, 25(2), 154-158. doi: 10.3109/09638237.2015.1101414

Boyatzis, R. . (1998). Transforming Qualitative Information. Thousand Oaks, CA: Sage Publications.

Braun, V. , \& Clarke, V. . (2006). Using thematic analysis in psychology. Qualitative Research in Psychology, 3(2), 77-101.

Bryant, A., \& Charmaz, K. (2010). The Sage Book of Grounded Theory. London: Sage.

Canavan, C., West, J., \& Card, T. (2014). The epidemiology of irritable bowel syndrome. Clin Epidemiol, 6, 71-80. doi: 10.2147/CLEP.S40245

Chilcot, J., \& Moss-Morris, R. (2013). Changes in illness-related cognitions rather than distress mediate improvements in irritable bowel syndrome (IBS) symptoms and disability following a brief cognitive behavioural therapy intervention. Behaviour Research and Therapy, 51(10), 690-695.

Creswell, J. W. . (2003). Research design. Qualitative, quantitative, and mixed methods approaches. (C. S. Thousand Oaks Ed. 2nd ed.).

De Vogli, R., Chandola, T., \& Marmot, M. G. (2007). Negative aspects of close relationships and heart disease. Arch Intern Med, 167(18), 1951-1957. doi: 10.1001/archinte.167.18.1951

Deary, V., Chalder, T., \& Sharpe, M. (2007). The cognitive behavioural model of medically unexplained symptoms: a theoretical and empirical review. Clin Psychol Rev, 27(7), 781-797. doi: 10.1016/j.cpr.2007.07.002

Dey, I. . (1993). Qualitative data analysis: a user friendly guide for social scientists. . London: Routeledge.

Drossman, D. A. (1998). Presidential address: Gastrointestinal illness and the biopsychosocial model. Psychosom Med, 60(3), 258-267.

Drossman, D. A. (2006). The functional gastrointestinal disorders and the Rome III process. Gastroenterology, 130(5), 1377-1390. doi: 10.1053/j.gastro.2006.03.008

Drossman, D. A., Creed, F. H., Olden, K. W., Svedlund, J., Toner, B. B., \& Whitehead, W. E. (1999). Psychosocial aspects of the functional gastrointestinal disorders. Gut, 45 Suppl 2, II25-30.

Drossman, D. A., Leserman, J., Li, Z., Keefe, F., Hu, Y. J., \& Toomey, T. C. (2000). Effects of coping on health outcome among women with gastrointestinal disorders. Psychosom Med, 62(3), 309317. 
Engel, G. L. (1980). The clinical application of the biopsychosocial model. Am J Psychiatry, 137(5), 535-544. doi: 10.1176/ajp.137.5.535

Everitt, H., Landau, S., Little, P., Bishop, F. L., McCrone, P., O'Reilly, G., .. team, Actib trial. (2015). Assessing Cognitive behavioural Therapy in Irritable Bowel (ACTIB): protocol for a randomised controlled trial of clinical-effectiveness and cost-effectiveness of therapist delivered cognitive behavioural therapy and web-based self-management in irritable bowel syndrome in adults. BMJ Open, 5(7), e008622. doi: 10.1136/bmjopen-2015-008622

Fava, G.A. , Freyberger, H.J. , \& Bech, P. . (1995). Diagnostic criteria for use in psychosomatic research. Psychothery Psychosomatics, 63, 1-8.

Fond, G., Loundou, A., Hamdani, N., Boukouaci, W., Dargel, A., Oliveira, J., .. Boyer, L. (2014). Anxiety and depression comorbidities in irritable bowel syndrome (IBS): a systematic review and meta-analysis. Eur Arch Psychiatry Clin Neurosci, 264(8), 651-660. doi: 10.1007/s00406014-0502-z

Francis, C. Y., Morris, J., \& Whorwell, P. J. (1997). The irritable bowel severity scoring system: a simple method of monitoring irritable bowel syndrome and its progress. Aliment Pharmacol Ther, 11(2), 395-402.

Grodzinsky, E., Walter, S., Viktorsson, L., Carlsson, A. K., Jones, M. P., \& Faresjo, A. (2015). More negative self-esteem and inferior coping strategies among patients diagnosed with IBS compared with patients without IBS--a case-control study in primary care. BMC Family Practice, 16(6), 28.

Gross, J. J. (2002). Emotion regulation: affective, cognitive, and social consequences. Psychophysiology, 39(3), 281-291. doi: 10.1017.S0048577201393198

Hauser, G., Pletikosic, S., \& Tkalcic, M. (2014). Cognitive behavioral approach to understanding irritable bowel syndrome. World J Gastroenterol, 20(22), 6744-6758. doi: 10.3748/wjg.v20.i22.6744

Hawkins, K. A., \& Cougle, J. R. (2011). Anger problems across the anxiety disorders: findings from a population-based study. Depress Anxiety, 28(2), 145-152. doi: 10.1002/da.20764

Henningsen, P., Zimmermann, T., \& Sattel, H. (2003). Medically unexplained physical symptoms, anxiety, and depression: a meta-analytic review. Psychosom Med, 65(4), 528-533.

Hewitt, P. L., Flett, G. L., Besser, A., Sherry, S. B., \& McGee, B. (2003). Perfectionism is multidimensional: a reply to Shafran, Cooper and Fairburn. Behav Res Ther, 41(10), 12211236.

Jaeger, M. E., \& Rosnow, R. L. . (1988). Contextualism and its implications for psychological inquiry.

. British Journal of Psychology, 79, 63-75.

Joffe, H. , \& Yardley, L. . (2004). In D. Marks \& L. Yardley (Eds.), Research Methods for Clinical and Health Psychology (pp. 56-68). London: Sage.

Jones, M., Koloski, N., Boyce, P., \& Talley, N. J. (2011). Pathways connecting cognitive behavioral therapy and change in bowel symptoms of IBS. Journal of Psychosomatic Research, 70(3), 278-285. doi: DOI 10.1016/j.jpsychores.2010.10.004

Kabra, N., \& Nadkarni, A. (2013). Prevalence of depression and anxiety in irritable bowel syndrome: A clinic based study from India. Indian J Psychiatry, 55(1), 77-80. doi: 10.4103/00195545.105520

Kempke, S., Luyten, P., Mayes, L. C., Van Houdenhove, B., \& Claes, S. (2016). Self-critical perfectionism predicts lower cortisol response to experimental stress in patients with chronic fatigue syndrome. Health Psychol, 35(3), 298-307. doi: 10.1037/hea0000299

Knowles, S. R., Austin, D. W., Sivanesan, S., Tye-Din, J., Leung, C., Wilson, J., . . Hebbard, G. (2016). Relations between symptom severity, illness perceptions, visceral sensitivity, coping strategies and well-being in irritable bowel syndrome guided by the common sense model of illness. Psychol Health Med, 1-11. doi: 10.1080/13548506.2016.1168932

Lackner, J. M., Gudleski, G. D., Firth, R., Keefer, L., Brenner, D. M., Guy, K., . . Sitrin, M. D. (2013). Negative aspects of close relationships are more strongly associated than supportive 
personal relationships with illness burden of irritable bowel syndrome. J Psychosom Res, 74(6), 493-500. doi: 10.1016/j.jpsychores.2013.03.009

Lackner, J. M., Jaccard, J., Krasner, S. S., Katz, L. A., Gudleski, G. D., \& Blanchard, E. B. (2007). How does cognitive behavior therapy for irritable bowel syndrome work? A mediational analysis of a randomized clinical trial. Gastroenterology, 133(2), 433-444. doi: DOI 10.1053/j.gastro.2007.05.014

Le, H. N., Berenbaum, H., \& Raghavan, C. (2002). Culture and alexithymia: mean levels, correlates, and the role of parental socialization of emotions. Emotion, 2(4), 341-360.

Ljotsson, B., Hesser, H., Andersson, E., Lindfors, P., Hursti, T., Ruck, C., . . Hedman, E. (2013). Mechanisms of change in an exposure-based treatment for irritable bowel syndrome. $J$ Consult Clin Psychol, 81(6), 1113-1126. doi: 10.1037/a0033439

Lovell, R. M., \& Ford, A. C. (2012). Global prevalence of and risk factors for irritable bowel syndrome: a meta-analysis. Clin Gastroenterol Hepatol, 10(7), 712-721 e714. doi: 10.1016/j.cgh.2012.02.029

Madill, A., Jordan, A. , \& Shirley, C. (2000). Objectivity and reliability in qualitative analysis: Realist, contextualist and radical constructionist epistemologies. . British Journal of Psychology, 91, $1-20$.

Manning, A. P., Thompson, W. G., Heaton, K. W., \& Morris, A. F. (1978). Towards positive diagnosis of the irritable bowel. Br Med J, 2(6138), 653-654.

Nellesen, D., Yee, K., Chawla, A., Lewis, B. E., \& Carson, R. T. (2013). A systematic review of the economic and humanistic burden of illness in irritable bowel syndrome and chronic constipation. J Manag Care Pharm, 19(9), 755-764. doi: 10.18553/jmcp.2013.19.9.755

Phillips, K., Wright, B. J., \& Kent, S. (2013). Psychosocial predictors of irritable bowel syndrome diagnosis and symptom severity. Journal of Psychosomatic Research, 75(5), 467-474. doi: DOI 10.1016/j.jpsychores.2013.08.002

Porcelli, P., De Carne, M., \& Leandro, G. (2014). Alexithymia and gastrointestinal-specific anxiety in moderate to severe irritable bowel syndrome. Comprehensive Psychiatry, 55(7), 1647-1653.

Porcelli, P., De Carne, M., \& Leandro, G. (2017). The role of alexithymia and gastrointestinal-specific anxiety as predictors of treatment outcome in irritable bowel syndrome. Comprehensive Psychiatry, 73, 127-135.

Pressman, S. D., \& Cohen, S. (2005). Does positive affect influence health? Psychol Bull, 131(6), 925971. doi: 10.1037/0033-2909.131.6.925

Rachman, S. (1980). Emotional Processing. Behaviour Research and Therapy, 18(1), 51-60. doi: Doi 10.1016/0005-7967(80)90069-8

Rachman, S. (2001). Emotional processing, with special reference to post-traumatic stress disorder. International Review of Psychiatry, 13(3), 164-171. doi: Doi 10.1080/09540260120074028

Reme, S. E., Stahl, D., Kennedy, T., Jones, R., Darnley, S., \& Chalder, T. (2011). Mediators of change in cognitive behaviour therapy and mebeverine for irritable bowel syndrome. Psychol Med, 41(12), 2669-2679. doi: 10.1017/S0033291711000328

Rimes, K. A., \& Chalder, T. (2010). The Beliefs about Emotions Scale: Validity, reliability and sensitivity to change. Journal of Psychosomatic Research, 68(3), 285-292. doi: DOI 10.1016/j.jpsychores.2009.09.014

Robles, T. F., Brooks, K. P., \& Pressman, S. D. (2009). Trait positive affect buffers the effects of acute stress on skin barrier recovery. Health Psychol, 28(3), 373-378. doi: 10.1037/a0014662

Sibelli, A., Chalder, T., Everitt, H., Workman, P., Windgassen, S., \& Moss-Morris, R. (2016). A systematic review with meta-analysis of the role of anxiety and depression in irritable bowel syndrome onset. Psychol Med, 1-16. doi: 10.1017/S0033291716001987

Sirois, F. M., \& Molnar, D. S. (2014). Perfectionism and maladaptive coping styles in patients with chronic fatigue syndrome, irritable bowel syndrome and fibromyalgia/arthritis and in healthy controls. Psychother Psychosom, 83(6), 384-385. doi: 10.1159/000365174 
Spence, M. J., \& Moss-Morris, R. (2007). The cognitive behavioural model of irritable bowel syndrome: a prospective investigation of patients with gastroenteritis. Gut, 56(8), 1066 1071. doi: DOI 10.1136/gut.2006.108811

Spiller, R., Aziz, Q., Creed, F., Emmanuel, A., Houghton, L., Hungin, P., . . Clinical Services Committee of The British Society of, Gastroenterology. (2007). Guidelines on the irritable bowel syndrome: mechanisms and practical management. Gut, 56(12), 1770-1798. doi: 10.1136/gut.2007.119446

Talley, N. J., Holtmann, G., \& Walker, M. M. (2015). Therapeutic strategies for functional dyspepsia and irritable bowel syndrome based on pathophysiology. J Gastroenterol, 50(6), 601-613. doi: 10.1007/s00535-015-1076-x

Tanaka, Y., Kanazawa, M., Fukudo, S., \& Drossman, D. A. (2011). Biopsychosocial model of irritable bowel syndrome. J Neurogastroenterol Motil, 17(2), 131-139. doi: 10.5056/jnm.2011.17.2.131

Taylor, G. J. (1984). Alexithymia - Concept, Measurement, and Implications for Treatment. American Journal of Psychiatry, 141(6), 725-732. 